\section{Maloka}

\section{Inspiración e innovación empresarial en una sola palabra}

\author{
Lina María Echeverri Cañas
}

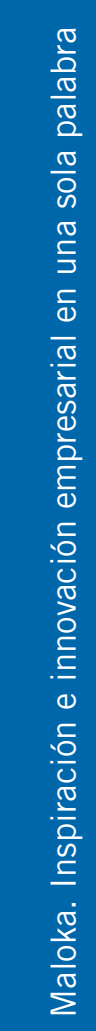

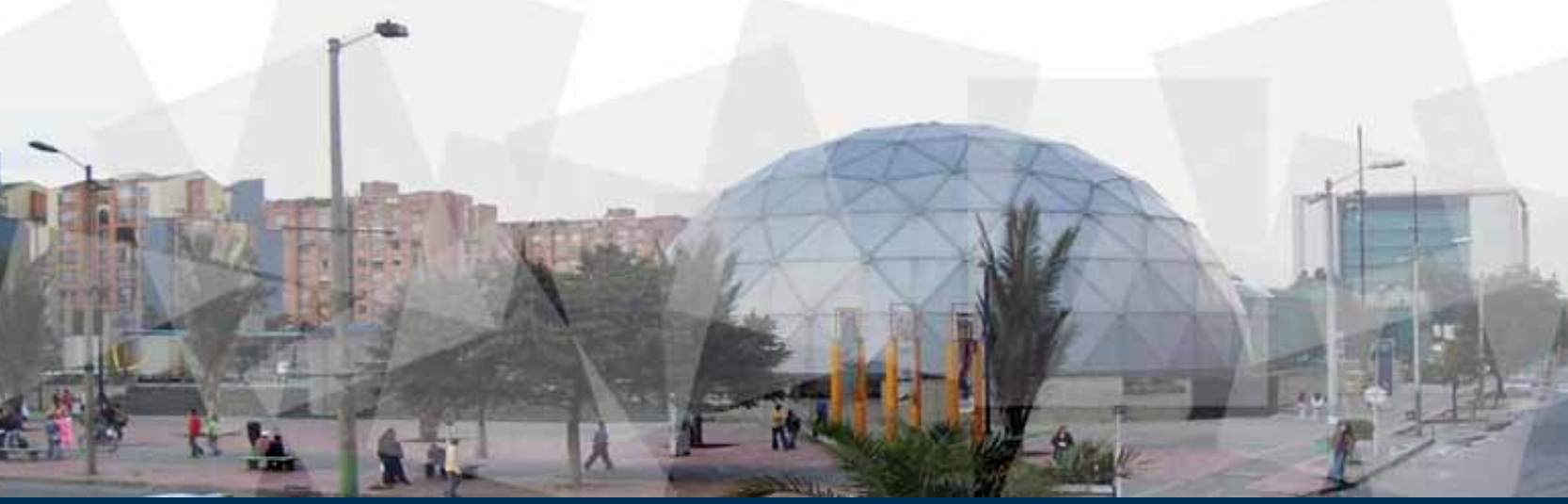

Lina Maria Echeverri Cañas

Profesional en Desarrollo Familiar de la Universidad de Caldas, Economista Empresarial de la Universidad Autónoma de Manizales y Magistra en Administración con énfasis en Mercadotecnia del Instituto Tecnológico de Estudios Superiores de Monterrey ITESM. Secretaria General del CESA y Profesora investigadora en el área de mercadeo, investigación de mercados y comportamiento del consumidor en pregrado y postgrado. Con una experiencia de 10 años en consultoría empresarial y docencia, en el área de investigación de mercados y diseño de planes de mercadeo. Fue coordinadora del programa de Economía Empresarial y de la Especialización en Negocios y Marketing Internacional de la Universidad Autónoma de Manizales.

Ha desarrollado diversas investigaciones y publicaciones en temas administrativos y de mercadeo. Su último libro publicado por editorial nacional e internacional se titula "Marketing práctico: una visión estratégica de un plan de mercadeo". Es coautora del caso académico de "Colombia es pasión".

También ha sido profesora invitada por las Cámaras de Comercio de Manizales y Pereira, Universidad Nacional de Colombia, Universidad del Rosario, La Salle College, Universidad Autónoma de Manizales e INCOLDA. 
El Colegio de Estudios Superiores de Administración CESA es una entidad privada, sin ánimo de lucro, que fue fundada en 1974 en Bogotá por un grupo de empresarios y figuras nacionales liderados por los doctores Carlos Lleras Restrepo y Hernán Echavarría Olózaga, con el apoyo del Instituto Colombiano de Administración, INCOLDA y de la Asociación Nacional de Industriales, ANDI.

El CESA es una institución universitaria que aglutina a los miembros de su comunidad alrededor del propósito de formar profesionales de la Administración de Empresas, que a la vez sean líderes empresariales, mediante la transmisión de experiencias, conocimientos y valores.

www.cesa.edu.co
Maloka más que un centro interactivo, es la materialización de un sueño que ha logrado convertirse en un ícono nacional en lo referente a la Apropiación de Ciencia y Tecnología en Colombia. El verdadero significado de Maloka está representado en que la educación y el conocimiento construyen una nación. Su modelo de negocio innovador es expuesto a través de un caso académico que tiene como propósito presentar las experiencias, los logros, las limitaciones y el crecimiento de una organización muy particular.

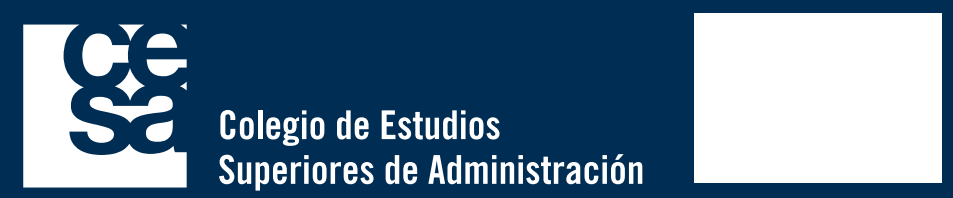




\section{MALOKA}

\section{INSPIRACIÓN E INNOVACIÓN EMPRESARIAL EN UNA SOLA PALABRA}

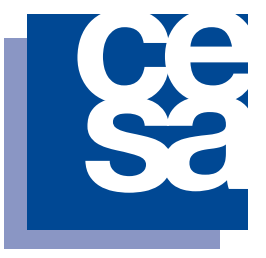

Colegio de Estudios

Superiores de Administración 


\title{
MALOKA \\ INSPIRACIÓN E INNOVACIÓN EMPRESARIAL EN UNA SOLA PALABRA
}

\author{
Lina María Echeverri Cañas
}


PRIMERA EDICIÓN, BOGOTÁ, D.C., ABRIL DE 2010

COORDINACIÓN EDITORIAL: DEPARTAMENTO DE COMUNICACIONES Y MARKETING CESA

CORRECCIÓN DE ESTILO: MARÍA JOSÉ DÍAZ GRANADOS

DIAGRAMACIÓN Y DISEÑO: DIEGO ESTEBAN ROMERO VARÓN IMPRESIÓN:

C 2010 COlegio de Estudios Superiores de AdMinistración CESA

(C) 2010 LINA MARÍA ECHEVERRI CAÑAS

ISBN: 978-958-97648-3-1

COMUNICACIONES

CRA. 6 NO. 35 - 28 CASA LLERAS

COMUNICACIONES@CESA.EDU.CO

TODOS LOS DERECHOS RESERVADOS.

ESTA OBRA NO PUEDE SER REPRODUCIDA SIN EL PERMISO PREVIO ESCRITO.

IMPRESO Y HECHO EN COLOMBIA

PRINTED AND MADE IN COLOMBIA 


\section{REGISTRO BIBLIOGRÁFICO:}

ECHEVERRI CAÑAS, LINA MARÍA. MALOKA: INSPIRACIÓN E INNOVACIÓN EMPRESARIAL EN UNA SOLA PALABRA. BOGOTÁ: CESA, 2010. 58P.

\section{DESCRIPTORES:}

MALOKA (BOGOTA)- COLOMBIA-ESTUDIO DE CASOS / INNOVACIONES TECNOLOGICAS-COLOMBIA-ESTUDIO DE CASOS / ADMINISTRACION DE EMPRESASCOLOMBIA-ESTUDIO DE CASOS. 


\section{CONTENIDO}

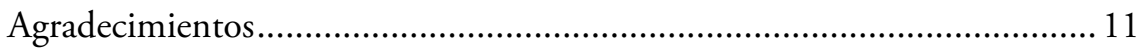

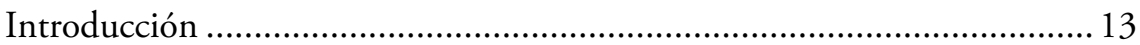

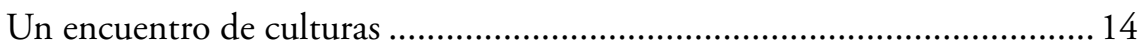

El primer paso fue el sueño..................................................................... 15

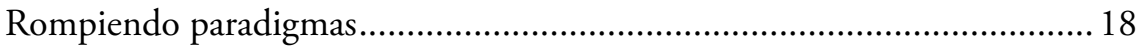

El final de un sueño y el comienzo de una realidad .................................... 24

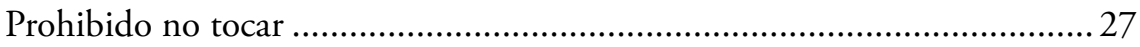

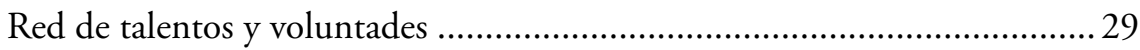

Una nueva forma de democratizar la educación .......................................... 31

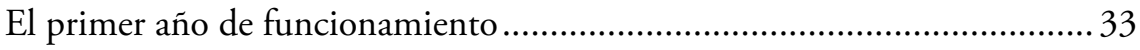

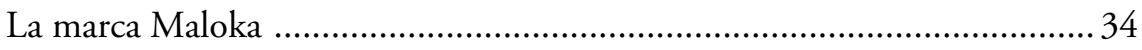

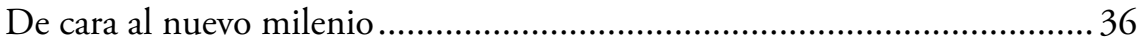

Más imaginación en medio de la crisis........................................................ 40

Maloka, el camino a la esperanza............................................................... 44

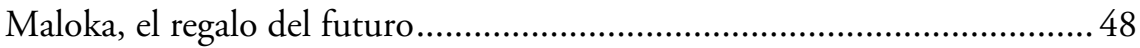

Hacia la renovación y expansión de Maloka................................................ 51

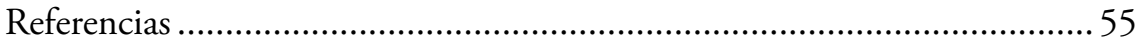

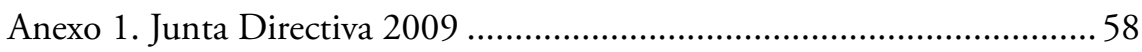

Anexo 2. Promotores y patrocinadores ……….......................................... 59 


\section{AGRADECIMIENTOS}

uiero manifestar mi agradecimiento al grupo ejecutivo de Maloka, en
cabeza de la Directora, doctora Nohora Elízabeth Hoyos; al presiden-
te de la Asociación Colombiana para el Avance de la Ciencia, doctor
Eduardo Posada; al Rector del CESA, doctor José Manuel Restrepo, y a quienes
de manera directa e indirecta contribuyeron al resultado de un caso académico
que tiene como propósito integrar la historia y el desarrollo de una experiencia
significativa sobre la Corporación Maloka.
Merecen un agradecimiento especial: Samanta Arango, Subdirectora Co-
mercial y de Mercadeo de Maloka; Adriana Rivera, Coordinadora Jurídica de
Maloka; César Nieto, Subdirector Administrativo y Financiero; los asistentes
de investigación: Marcela Andrade y Jaime Bonilla, estudiantes de la Especiali-
zación en Mercadeo Estratégico del CESA por su compromiso, interés y apoyo
en la construcción del caso Maloka. 


\section{INTRODUCCIÓN}

\section{"CREO QUE EN LA ALEGRÍA, EN EL AMOR Y EN EL COMPROMISO SOCIAL HACIA LA INNOVACIÓN, ESTÁ EL SIGNIFICADO DE MALOKA"}

\section{NOHORA ELÍZABETH Hoyos, Directora de MALOKA}

$\mathbf{E}$ scribir sobre un sueño parece simple. Pero cuando el sueño proviene de un imaginario colectivo, con un alto compromiso hacia la sociedad colombiana en temas de ciencia y tecnología, es mucho más complejo.

Escuchar la historia sobre la creación de la Corporación Maloka en las voces de sus pioneros y, simultáneamente, sentir la emoción que nos envuelve de principio a fin, es una experiencia invaluable. Maloka, más allá de un modelo empresarial, representa una nueva forma de apropiar conocimiento.

El siguiente caso académico pretende dar un acercamiento a las vivencias, experiencias y sensaciones de una organización muy particular. Una idea que fue cuestionada en sus inicios y, a su vez, defendida por un grupo de pioneros que acertaron en el diseño y la construcción de un innovador modelo pedagógico.

El estudio de caso nace de una iniciativa conjunta entre la Corporación Maloka y la línea de investigación en mercadeo del CESA, cuya intencionalidad es hacer el levantamiento de un caso académico sobre el Centro Interactivo.

El estudio de caso es la descripción de una situación real de una empresa o una organización. Con el uso de los estudios de caso se mantiene la promesa de utilizar una técnica pedagógica que fomenta la socialización e interacción de los estudiantes y docentes porque humaniza la ciencia y se constituye en una forma didáctica que ilustra la metodología y los valores científicos. A través de los estudios de caso, el estudiante desarrolla competencias en términos de aprendizaje problemático y pensamiento crítico.

En el proceso de enseñanza-aprendizaje se ha encontrado que el estudio de caso flexibiliza la apropiación de conocimientos. Con esta estrategia se recogen experiencias y situaciones que dirigen al lector hacia el conocimiento de un fenómeno cuyo abordaje rompe con los esquemas de enseñanza tradicionales. 